
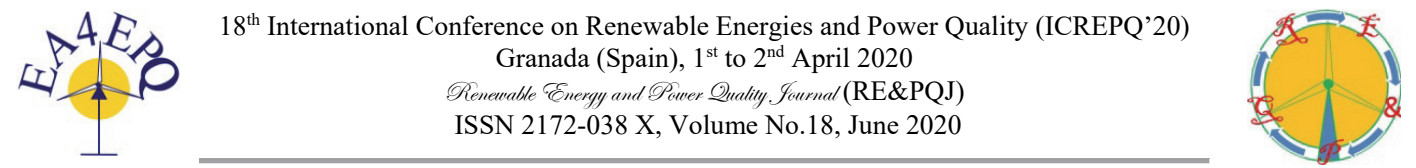

\title{
Analysing renewable energy flow distribution and its influence on grid electricity prices
}

\author{
Vladislav O. Samoylenko ${ }^{1}$, Andrew V. Pazderin ${ }^{1}$ and Sergei A. Bychkov ${ }^{2}$ \\ ${ }^{1}$ Department of Automated Electrical Systems \\ Ural Federal University \\ Mira str., 19, 620002 Yekaterinburg (Russia) \\ Phone/Fax number: +007 343 359-16-15, e-mail: vvsamoylenko@yandex.ru, a.v.pazderin@urfu.ru \\ ${ }^{2}$ Ural Power Engineering Institute \\ Ural Federal University \\ Mira str., 19, 620002 Yekaterinburg (Russia) \\ Phone/Fax number: +007 343 359-16-15, e-mail: s.a.bychkov@urfu.ru
}

\begin{abstract}
The paper presents an approach to the assessment of technical and economic efficiency for an electric energy (EE) transport in a distribution grid. It is focused on the extent of renewable energy sources (RES) integration efficiency according to energy prices in a grid. A two-step procedure for calculation is used in terms of the approach. The first one includes the obtaining of energy flows distribution in a grid. The next step provides the calculation of costs flows and nodal prices for electric energy. Both stages are performed using a developed branch flow model. Unlike power flow distribution describing the instant point of time, energy flow distribution developed for any operation duration deals with the integral values of the flows. This is of great importance for the deeply varying output profiles of the intermittent renewables. The model provides the direct utilization of electric energy meters data available in a grid. The approach presented is essential for locating the particular consumers of the renewable energy in terms of a grid's deep state and circuit diversity as well as for analyzing the influence of a renewable energy for electricity prices, and estimating the necessary renewables' levelised cost of energy (LCOE).
\end{abstract}

Key words. Power flow distribution, energy flow distribution, electric energy, LCOE, electricity prices.

\section{Introduction}

The steady-state parameters of a grid are typically obtained by carrying out the calculation of power flow distribution. It is conventionally performed using power flow equations based on the nodal voltages [1]. The power flow distribution describes the instant point of time. Due to the fact that persistent changes occur in power system, power flow distribution correctly represents the state in a grid when these changes are slow and their magnitude is insufficient. This feature limits the applicability of power flow distribution to short-time issues or to the issues concerned with the fit-and-forget approach, when the absolute minimum and maximum states are checked during power system design. Large power systems within relatively slow load changes of 3-5\% per hour historically enable the re-calculation of steady-state once in a hour
( $T=1 \mathrm{~h}$ ), although modern distribution grid requires to make it once in several minutes $T=5-15 \mathrm{~min}$ to represent the state correctly. In fact, a set of sequential noncorrelated and non-interrelated states of a fixed duration is computed in this case [2], [3]. The calculation is suitable for basic dispatch, scheduling and market purposes. Nevertheless, there are the cases when conventional approaches and models undergo serious methodical violations restricting the application of power flow distribution:

1) Due to the general nonlinearity of electric circuits (Joule-Lenz law, resistance variations, etc.), averaging over $T$ while deep and fast state changes occur leads to the strong qualitative errors in state representation [4]. The case is directly concerned with the intermittent renewables and low-voltage loads tending to rapid power fluctuations.

2) Topology diversity in grids of meshed structure and reverse electrical energy flow during $T$ leads to qualitative errors in state representation besides the quantative ones [5].

3) The prospective planning of any grid while considering the economic features to be based on some weighted and averaged parameters. There could be a set of the load curve profiles and generation output profiles available for the grid and their composition during the long periods is not always evident. A some integral estimation is needed.

4) EE prices may not be constant and vary at the commercial intervals that are not coincident with the dispatch intervals of fixed $T$.

5) The problem of steady-state calculation using measurements data is considered applying state estimation theory. For conventional state estimation, SCADA telemetry with quasi-instant power values is used. Energy management 
systems to provide the EE values for several minutes or hours. At the level of low-voltage systems, especially if electricity measurements are manually gathered from non-smart meters, the interval can be a month or even a year.

6) Besides the time-scale problems, the models for power flow distribution are typically sensitive for a grid scale. Zero and close to zero resistances of branches complicate the formation of conductivity matrix and observation of the computation results.

Renewable energy sources usually are interconnected to distribution systems where all the listed problem features are typical and should be taken into account. The detailed analysis of energy utilization from a renewable energy source and its influence on a grid nodal prices requires more specified models providing stable calculation in terms of deep state, topology and time-scale diversity, like branch-flow models [6], [7]. In fact, recent studies about branch flow models are concerned with the computational algorithm itself [8]-[10], and not the sphere the model application like distribution systems, distributed generation and RES in particular.

The aim of the paper is to adapt a branch-flow model to economic estimation of the renewables efficiency integration into electric grid. The results of the estimation are suitable for billing, markets, and prospective planning of distribution systems development. The model is presented further.

\section{Theoretical background}

\section{A. Energy flow distribution model}

The model for energy flow distribution in a grid to possess the following features: integral, providing grid topology changes accounting, scale-insensitive for time and grid length. These features are mostly essential for the branch flow model described by Energy Flow Problem solution [11]-[13]. The main input data for the EFP are the measurements of active and (optional) reactive EE. The result of this problem solution is balanced EE flows and losses distribution at all the elements of the electrical grid called Energy Flow Distribution (EFD).

The equations for EFP based on the EE balance equations are set for all the nodes and the branches of a grid. The equations of the nodal EE balances are set separately for active and reactive flows:

$$
\begin{aligned}
& \sum_{j \in \omega_{i}} W_{i j}^{p}+W_{i}^{p}+\Delta W_{i}^{p}=0 ; \\
& \sum_{j \in \omega_{i}} W_{i j}^{q}+W_{i}^{q}+\Delta W_{i}^{q}=0,
\end{aligned}
$$

where $W^{p}{ }_{i}, W^{q}{ }_{i}$ are the injections of active and reactive EE for node $i ; W^{p} i, W_{i j}^{q}$ are active and reactive EE flows across all the branches between nodes $i$ and $j ; \omega_{i}$ is the set of nodes incident to the node $i ; \Delta W^{p}{ }_{i}, \Delta W^{q}{ }_{i}$ are the losses of active and reactive EE in the node $i$ shunt; $N$ - is the number of model nodes.

The balance of EE in the branches is determined by $W_{i j}$ EE flow at the beginning of the branch, $W_{j i}$ at its end, and by the EE losses $\Delta W_{i j}$ :

$$
\begin{aligned}
& W_{i j}^{p}+W_{j i}^{p}+\Delta W_{i j}^{p}=0 ; \quad i j=1,2, \ldots M, \\
& W_{i j}^{q}+W_{j i}^{q}+\Delta W_{i j}^{q}=0,
\end{aligned}
$$

where $M-$ is the number of model branches. The balance equations (1) - (1) form the basis of the EFP state equations and they are linear.

Modelling the losses is discussed in details in [13] making the problem formulation linear or nonlinear. The most popular approaches are decremental losses accounting for linear estimation and Joule-Lenz law calculation for non-linear iteration-based estimation.

The model provides the direct electricity meters measurements data utilization. Due to the fact that all the measurements contain errors because of the non-ideal accuracy of metering complexes, a state estimation approaches to be used for obtaining the balanced energy flow distribution [14]. The weighted sum of flows deviation squares is used multiplied by meters accuracy coefficients:

$$
F=\sum_{i=1}^{K} \frac{1}{\left(\delta_{i}^{\%} \hat{W}_{i}\right)^{2}}\left(\hat{W}_{i}-W_{i}\right)^{2} \rightarrow \mathrm{min},
$$

where $\widehat{W}_{i}$ is the measured value for each considered flow.

The advantages of the model proposed are:

1) Energy flow distribution can be calculated in a single procedure for any time interval.

2) The equations of EE balances don't have residuals due to grid state and topology variations over the time $T$, that is, they always retain the relevance to the simulation [15], [16].

3) The flow-based model is insensitive to the scale of the equivalent circuit parameters. The conductivity is not used in calculations. It is possible to calculate the circuits containing zero resistance of the branches. The last EFP advantage is very important, since it allows to introduce in the equivalent circuit some intersection circuit breakers that are often equipped with EE meters.

4) Simplicity of the measurements' equations provides the calculation approximately five times faster than SE using voltages [15], [16].

The limitations of the model include the necessity to know the particular duration of switch off mode for any circuit component. Otherwise, it may results in a sufficient garble in energy flows outlay. It could be controlled for the branches of a grid circuit where the metering data are available. 


\section{B. Cost flow distribution model}

The idea of the cost flow distribution is that all the electric energy flows have cost depending upon electricity prices. Electricity prices depend upon power generation prices like LCOE [17], [18], as well as transmission and distribution (T\&D) prices. It is related to the set of market models [19]-[21] using the nodal prices, but has the following strong differences:

1) The cost flow model based on the energy flow distribution referred at $A$ section of the paper. It is assumed that the energy flows for nodes and branches as well as losses are calculated by the energy flow model and can be considered as absolutely balanced flows. The values to be calculated for an analyzed time interval, for example one month or one year. If a time interval under investigation is a retrospective interval, then the data from energy meters to be used as a basis for the energy flow model. To the contrary, if a prospective is considered, than forecasted values of energy input and output to be used.

2) The initial data can be presented directly by the capital costs of grid construction, if necessary. Power generation prices and especially T\&D prices are strongly influenced by the capital costs of their construction. Distinguishing grid market models provide that technical interconnection may be charged in a direct single-time payment or by the capital deprecation charges increasing the T\&D price and the resulting nodal electricity price. Both types of the initial data can be mixed in the model [22], [23].

In the model presented the branches of a grid circuit are associated with power lines, nodes are associated with substations' buses. The model corresponds to process of EE transport in the form of directed energy flows graph and directed cost flow graph. The process of both energy and cost flows allocation to be performed using the same grid equivalent circuit. The idea of transport costs distribution implies a sequence of transferring power generation costs and T\&D costs with respect to a path of EE flow direction in a grid graph. As a result, cost flows for each branch and each node can be calculated. It is useful to represent the process in the form of cost flows having the same direction as energy flows.

The nodal cost injections can be calculated as:

$$
C_{i}=c_{i} W_{i}
$$

where $c_{i}$ is the price of generation, $W_{i}$ is the EE output value. The nodal cost can be calculated as:

$$
C_{i \Sigma}=C_{i}+C_{i i}
$$

where $C_{i}$ is the nodal cost injection and $C_{i i}$ is the sum of the cost flows incoming to the node. Generally, the following cases are possible:
1) The node is an energy source node, then the flow inputs into the branches exporting the energy from the node proportionally to EE flows with respect to the total energy source output.

2) The node is a transit node of zero nodal injection, then the flow sum incoming the node distributes across the branches exporting the energy from the node proportionally to EE flows with respect to the transit nodal energy input.

3) The node is an terminal node of a radial grid. The nodal cost is equal to the sum of inflows.

4) The node is a transit node of non-zero nodal flow like energy source or load. Then the required nodal costs contain both nodal and transit flow components.

The nodal costs inflow into the adjacent branches proportionally to outcoming energy flows. Cost flow for the beginning of the branch adjacent to $i$ node are calculated as:

$$
C_{i j}=\frac{W_{i j}}{W_{i}+W_{i i}} \cdot \frac{1}{C_{i}+C_{i i}},
$$

where $W_{i i}$ is the sum of EE flows in branches adjacent to the $i$ node. The link between the cost at the beginning $C_{i j}$ and the end $C_{j i}$ of the branch:

$$
C_{j i}=C_{i j}+\Delta C_{i j}
$$

where $\Delta C_{i j}$ is the additional cost depending upon the model. If energy losses $\Delta W_{i j}$ are considered in a classical nodal prices market formulation, then:

$$
\Delta C_{i j}=C_{i j} \cdot \Delta W_{i j} / W_{i j}
$$

Generally, there can be a T\&D tariff according to the market model. In this case:

$$
\Delta C_{i j}=p_{T \& D} \cdot \Delta W_{i j}
$$

The process of flow costs distribution is accomplished until flow costs pass across a grid equivalent circuit to output nodes and there are no other cost flows paths. It is worth mentioning, that when the procedure of flow costs allocation is completed, total utility transport costs are exactly equal to a sum of flow costs in output nodes of a grid. The model implies that a total costs for $\mathrm{EE}$ is distributed between $N$ nodes and $M$ branches of a grid proportionally to T\&D costs. So, while EE is distributed through a grid, the cost per $\mathrm{kWh}$ increases.

\section{Analysing Renewable Energy flow distribution}

The aim of the model applied to RES analysis is to estimate their true integral influence on the nodal prices and prices of electrical energy flowing in a grid. According to $A$ Section of the paper the intermittent energy sources to be estimated by integral models, especially if a deep reverse of energy flows is possible in 
a distribution. According to $B$ Section of the paper, there initial data for energy consumption by load and existing generation is efficient to obtain using meters data. The initial data for the renewables output itself may be based on the scaled retrospective. Otherwise, any forecast output for the period under consideration to be used.

The analysis of the cost for renewables may be done in following ways:

1) Most of the manufacturers provide the information about the level of $\angle C O E$ for the particular model of the renewable energy source. It can be used as the initial rough approximation for the considered source.

2) Generally, $L C O E$ of renewables is a sophisticated function of the output $L C O E=f(W)$ to be matched with the forecasted output [24]-[26].

The algorithm of RES influence estimation includes a twostage procedure:

1) Calculation of the energy flow distribution;

2) Calculation of the cost flow distribution.

The workflow chart of the algorithm is presented in Fig. 1.

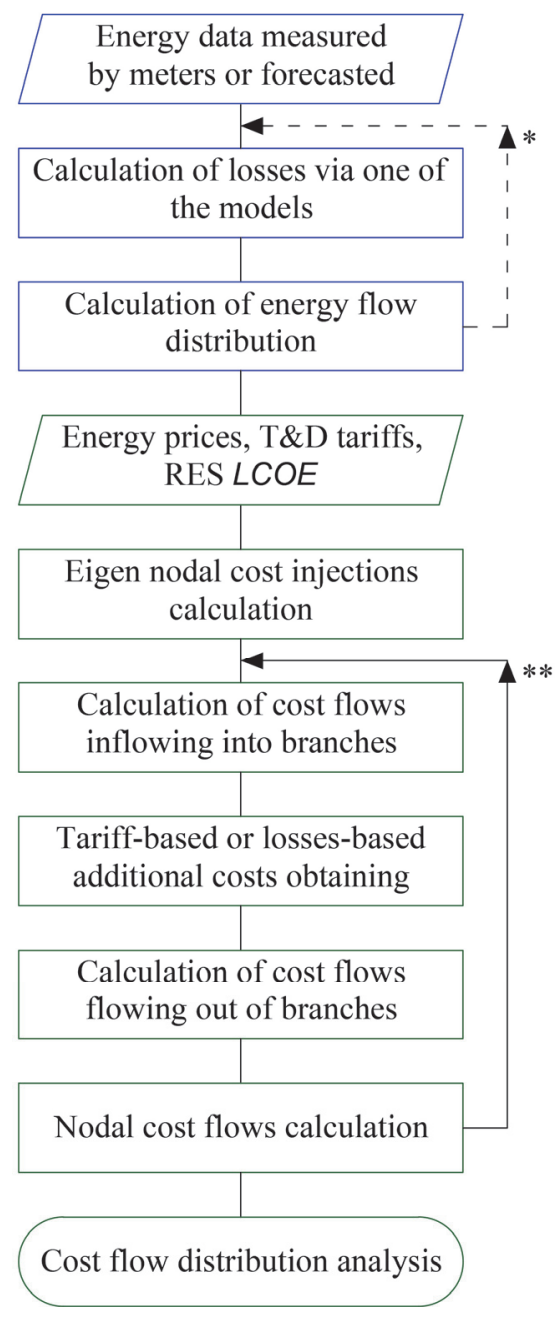

Fig. 2. The two-stage procedure of energy and cost flow distribution. Notes: *iterative procedure for non-linear losses only; **starts with the dead-end power lines and calculated for a grid until there are no other cost flows paths
In case of the reverse approach, when the required $L C O E$ to be obtained depending upon the energy flow distribution, the whole procedure of cost flow distribution turns out to be iterative.

Although the proposed model is insensitive for a number of nodes, general grid observability criteria to be met [13]. Otherwise, in case of a wide RES mix it turns out in equivalent $\angle C O E$ for a set of distinguishing RES that may compromise the efficiency estimation of particular sources. Also a high share of beyond-the-meter RES hides the real cost flow distribution if the output is not detected. Therefore, both kinds of problems to be eliminated by dedicated EE meters providing energy accounting and grid observability in terms of the model.

\section{Case Study}

The case study is conducted using real-prototype $10 \mathrm{kV}$ distribution grid (Fig. 2). Given the assumption of independent active and reactive flows distribution [11] just active EE flows are presented for the purposes of the clarification. The grid includes both radial and loop circuits. The transmission grid and power system are set as System equivalent at the first node. There are two types of energy consumers: residential households (that also may include some share of commercial and offices) and powerful industrial consumer. It is supposed that the consumer have its embedded beyond-the-meter generation, that's why it is considered as a prosumer. The corresponding energy output surplus is released to the grid during the periods of production shift changes.

Nodal EE values measured by the meters marked by " $\mathrm{X}$ " signs in Figure 2. The values for the purpose of clearness are preliminary shown for three periods of the day: offpeak $(a)$, semi-peak $(b)$ and peak $(c)$, correspondingly. The prices for EE are shown in Table I.

Table I. - Nodal electricity prices for basic/corrected renewables $L C O E$

\begin{tabular}{|l|c|c|c|c|}
\hline \multirow{2}{*}{ Time } & \multicolumn{4}{|c|}{ Price, $€$ per MWh } \\
\cline { 2 - 5 } & $\begin{array}{c}\text { Power } \\
\text { System } \\
\text { (PS) }\end{array}$ & $\begin{array}{c}\text { Industrial } \\
\text { Prosumer } \\
\text { (IP) }\end{array}$ & $\begin{array}{c}\text { Renewables } \\
\text { (Ren) }\end{array}$ & $\begin{array}{c}\text { Residential } \\
\text { (Rsid) }\end{array}$ \\
\hline Off-peak & 150.0 & 500.0 & $650.0 / 644.7$ & $368.5 / 366.9$ \\
\hline Semi-peak & 200.0 & $204.5 / 204.6$ & $650.0 / 644.7$ & $433.5 / 430.8$ \\
\hline Peak & 675.0 & $707.4 / 706.2$ & $650.0 / 644.7$ & $680.3 / 676.1$ \\
\hline Total & 514.5 & $658.7 / 657.5$ & $650.0 / 644.7$ & $517.6 / 514.5$ \\
\hline
\end{tabular}

It is supposed that the System is represented by bulk market typically obtaining the lowest EE price due to scale effect. The tasks for the calculation can be formulated as follows:

1) To calculate the integral nodal energy prices taking into account the variability and EE losses;

2) To determine the required $\angle C O E$ for the Renewables given the case when the cost for Residential can't exceed the price of the 
System' bulk market. This requirement is typical for some energy markets.

Energy flow distribution is calculated by the model presented at 2.A Section of the paper. The resulting energy features can be noted regarding the energy flow distribution:

1) There's a reverse energy flow in a loop circuit via Ren-IP power line. An attempt to average the flow and use a power flow distribution instead of the energy flow distribution leads to strong unbalances like it is described in Sections 1 and 2.A;

2) Both consumers supplied by three energy sources period of the day. An attempt to average the flow and use a power flow distribution instead of the energy flow distribution leads to the embedded generation unaccounted. In addition, in this case market mutual settlements become unclear.

Cost flow distribution is calculated by the model presented at 2.B Section of the paper. The following features can be noted regarding the energy flow distribution:

1) Due to the deep state diversity the distribution of the nodal prices is not evident before the cost flow calculation.

2) Weighted EE cost of 514,5 $€ / \mathrm{MWh}$ purchased from power system is closer to the peak price of $675 € / \mathrm{MWh}$;

3) Energy prosumer have the resulting cost of $658,7 € / \mathrm{MWh}$. There's a potential for increasing
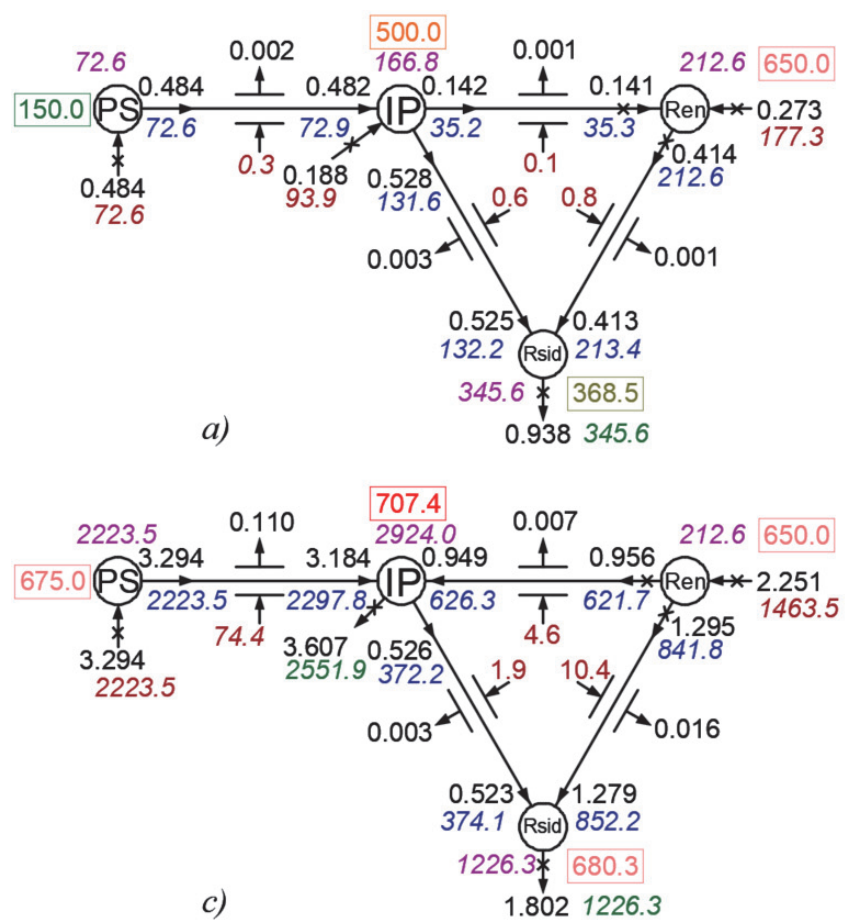
flow distribution is shown at Figure 2, d. The following establishing different combinations depending on

its embedded generation output due to the fact that there's a gap between the generation price of $500 € / \mathrm{MWh}$ and much more expensive EE purchased from the grid;

4) The cost of EE losses is only 2,1\% although the share of EE losses is 3,1\%;

5) The EE cost for Residential of $517,6 € / \mathrm{MWh}$ is close to the power system price of 514,5 $€ / \mathrm{MWh}$, although there are 2 energy sources much closer than power system.

The influence of Renewables' $L C O E$ is not the predominant due to the fact that there's also some share of EE to Residential distributed from Prosumer and System via the loop circuit. The solution of the (4)-(7) linear formulation by any solver gives the result that Renewables' $L C O E$ decrease to $644,7 € / \mathrm{MWh}$ provides the cost for Residential of 514,5 $€ / \mathrm{MWh}$ not exceeding the price of the System market. The corrected prices for this case are shown in Table I after the slash signs.

\section{Conclusion}

A two-stage procedure for estimation of energy flows and cost flows in a grid is presented. Both stages are performed based on the branch-flow model. It has the following features.

1. Unlike power flow distribution describing the instant point of time, energy flow distribution for any operation duration deals with the integral values of the flows. This is of great importance for the deeply varying output profiles of the intermittent renewables.
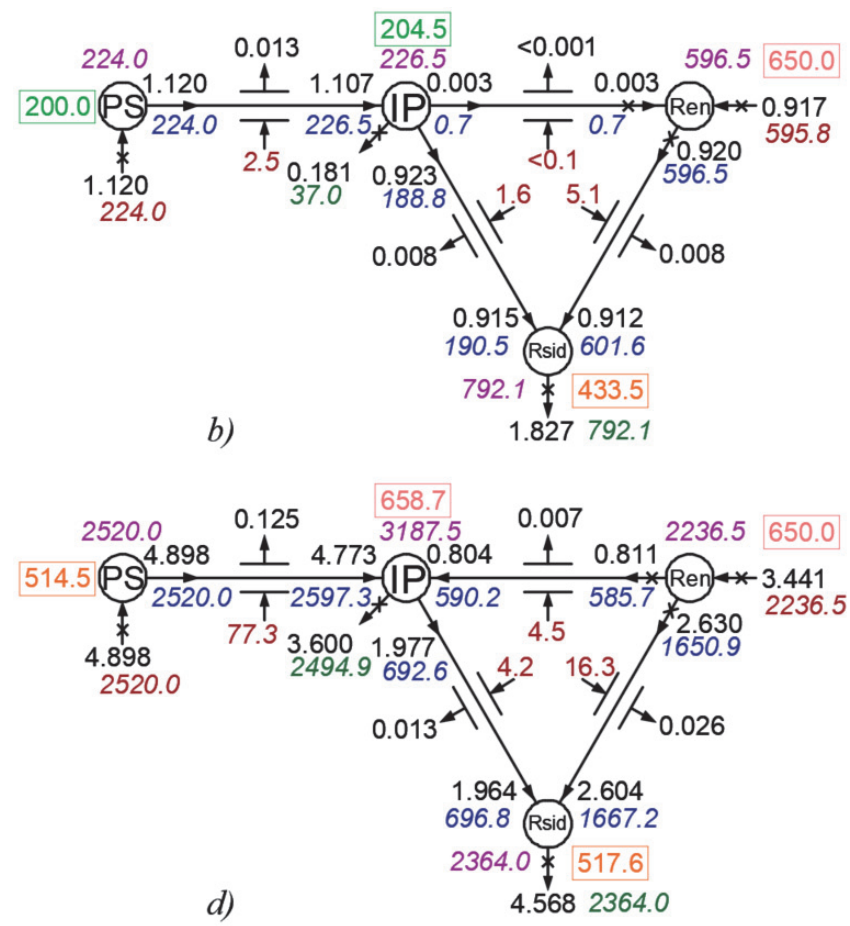

Fig. 2. Energy flow distribution and cost flow distribution per off-peak $(a)$, semi-peak $(b)$, peak $(c)$, and total per day $(d)$. Legend: electricity flow in MWh, nodal cost injection in $€$, nodal cost output in $€$, branch cost flow in $€$, nodal costs in $€$, [nodal price] in $€ / M W h$. The values of energy losses are shown with 0.001 accuracy and may not coincide due to the rounding 
2. The model provides the direct utilization of electric energy meters data available in a grid for well-fitted estimation.

3. The approach presented is essential for locating the particular consumers of the renewable energy in terms of a grid's deep state and circuit diversity as well as for analyzing the influence of a renewable energy for electricity prices, and estimating the necessary renewables $L C O E$ levels.

4. The model is sensitive for the duration of power lines and substation buses disconnection, so these events to be detected by Smart Meters directly or as energy flow absence.

Further investigations regarding the topic may include the optimization issue for renewables placement. It may be carried out using different criteria, like energy losses costs increments (penalty factors) and feed-in tariffs. Both criteria requires a modification of the two-stage procedure presented in the paper.

\section{Acknowledgement}

The work was supported by Act 211 Government of the Russian Federation, contract № 02.A03.21.0006.

\section{References}

[1] J.J. Grainger and W.D. Stevenson, Power system analysis, McGraw-Hill (1994), pp 787.

[2] B. Hayes and M. Prodanovic, "State Estimation Techniques for Electric Power Distribution Systems" (2014). 2014 European Modelling Symposium (EMS), Pisa, pp. 303-308.

[3] J. Chen, Y. Dong, and H. Zhang, "Distribution system state estimation: A survey of some relevant work", Proceedings of the $35^{\text {th }}$ Chinese Control Conference (2016), pp. 99859989.

[4] A.V. Pazderin, "Solving the problem of determining energy flows in an electric grid using the methods of state estimation" (2004). Electrichestvo, Vol. 12, pp. 2-7.

[5] A.V. Pazderin, "The problem of modeling the load flow in a grid" (2004). Electrichestvo, Vol. 10, pp 2-8.

[6] M. Farivar and S.H. Low, "Branch flow model: Relaxations and convexification - Part I" (2013). IEEE Transactions on Power Systems, Vol. 28, Issue 3, pp. 2554-2564.

[7] M. Farivar and S.H. Low, "Branch flow model: Relaxations and convexification - Part II" (2013). IEEE Transactions on Power Systems, Vol. 28, Issue 3, pp. 2565-2572.

[8] L.H. Macedo, C.V. Montes, and J.F. Franco et al., "MILP branch flow model for concurrent AC multistage transmission expansion and reactive power planning with security constraints" (2016). IET Generation, Transmission and Distribution, Vol. 10, Issue 12, pp. 3023-3032.

[9] J. Ren, P. He, and G. Li, "A new searching algorithm of power flow transferring for multi-branch removal event based on virtual-branch model and FTIL" (2017). Dianwang Jishu/Power System Technology, Vol. 41, Issue 1, pp. 194-200.

[10] W. Li, X. Han, and B. Zhang, "A closed format power flow algorithm based on branch models" (2012). Dianwang Jishu/Power System Technology, Vol. 36, Issue 3, pp. 113119.

[11] A.V. Pazderin, "The solution of power distribution problem for localization of commercial power losses" (2004) Promyshlennaya Energetika, Vol. 9, pp. 17-21.
[12] A. V. Pazderin, E. A. Plesniaev, and E. V. Mashalov, "An energy flow calculation method based on energy meters data" (2008).Proc. of the Third International Conference on Electric Utility Deregulation and Restructuring and Power Technologies, Nanjuing, pp. 834-839.

[13] A.V. Pazderin, S.E. Kokin, A.O. Egorov, and E.S. Kochneva, "Solution of energy flow problem using state estimation technique" (2009). Proc. of the Industrial Electronics Conference, pp. 1736-1741.

[14] E. A. Plesniaev and A. V. Pazderin, "Analysis of the Computation Techniques for Energy Flow Problem Solving" (2005). Proc. of The International Conference on "Computer as a Tool", Belgrade, pp. 1469-1472.

[15] M. E. Baran and A. W. Kelley, "A branch-current-based state estimation method for distribution systems" (2005). IEEE Transactions on Power Systems, Vol. 10, Issue 1, pp. 483-491.

[16] J. H. Teng, "Using voltage measurements to improve the results of branch-current-based state estimators for distribution systems" (2002). IEE Proceedings Generation, Transmission, Distribution, Vol. 149, Issue 6, pp. 667-672.

[17] L. Reichenberg, F. Hedenus, and M. Odenberger et al. "The marginal system LCOE of variable renewables Evaluating high penetration levels of wind and solar in Europe" (2018) Energy, Vol. 152, pp. 914-924.

[18] F. Ueckerdt, L. Hirth, and G. Luderer, "System LCOE: What are the costs of variable renewables?" (2013). Energy, Vol. 63, pp. 61-75.

[19] E. Bjørndal, M. Bjørndal, H. Cai, "Nodal pricing in a coupled electricity market" (2014). Proc. of the 11th International Conference on the European Energy Market, pp. $0-5$.

[20] P.M. Sotkiewicz and J.M. Vignolo, "Nodal pricing for distribution networks: efficient pricing for efficiency enhancing DG" (2006), IEEE Transactions on Power Systems, Vol. 21, Issue 2, pp. 1013-1014.

[21] R. K. Singh and S. K. Goswami, "Evaluation of Nodal Prices and Revenue of Distributed Generation in Distribution Network Including Load Model" (2008). Proc. of the Region 10 and the Third international Conference on Industrial and Information Systems, pp. 05.

[22] A.A. Pazderin, N.D. Mukhlynin, and A.V. Pazderin, "Technical and Economic Model of Energy Transmission and Distribution Based on the Smart Metering Technologies" (2017), Proc. of the 26th IEEE International Symposium on Industrial Electronics, Edinburgh, UK, pp. 163-168.

[23] A.A. Pazderin, A.V. Pazderin, and A.A. Firsova, "Energy-cost flows model of electric energy distribution at an electric network" (2017), The 11th IEEE International Conference on Compatibility, Power Electronics and Power Engineering. Cadiz, Spain, pp. 308-312.

[24] D.A. Sunter, B. Murray, and M. Lehmann, et al., "Twostage Monte Carlo simulation to forecast levelized cost of electricity for wave energy" (2017). Proc. of the 2017 IEEE 6th International Conference on Renewable Energy Research and Applications, pp. 0-5.

[25] J. A. Dzieża, "Stochastic LCOE in portfolio selection of electricity generation" (2017). Proc. of the 2017 IEEE International Conference on Environment and Electrical Engineering and 2017 IEEE Industrial and Commercial Power Systems Europe, pp. 0-5.

[26] C. Lucheroni, and C. Mari, "Stochastic LCOE for optimal electricity generation portfolio selection" (2014), Proc. of the 11th International Conference on the European Energy Market, pp. 0-5. 